\title{
Robust Generalized Total Least Squares Iterative Closest Point Registration
}

\author{
Raúl San José Estépar ${ }^{1}$, Anders Brun ${ }^{1,2}$, and Carl-Fredrik Westin ${ }^{1}$ \\ 1 Laboratory of Mathematics in Imaging, Brigham and Women's Hospital, \\ Harvard Medical School, Boston, MA, USA \\ $\{r j$ osest, anders, westin\}@bwh.harvard.edu \\ 2 Department of Biomedical Engineering, Linköping University, Sweden.
}

\begin{abstract}
This paper investigates the use of a total least squares approach in a generalization of the iterative closest point (ICP) algorithm for shape registration. A new Generalized Total Least Squares (GTLS) formulation of the minimization process is presented opposed to the traditional Least Squares (LS) technique. Accounting for uncertainty both in the target and in the source models will lead to a more robust estimation of the transformation. Robustness against outliers is guaranteed by an iterative scheme to update the noise covariances. Experimental results show that this generalization is superior to the least squares counterpart.
\end{abstract}

\section{Introduction}

The iterative closest point (ICP) algorithm [1] has been extensively used as optimization technique for rigid model based registration based on features extracted from medical datasets. ICP is an iterative descent procedure which seeks to minimize the sum of the squared distances between all points in a source and their closest points in a target model. As stated by Besl and McKay [1], ICP cannot deal with outliers and unequal uncertainty among points.

When there is a known correspondence between the points in the source and the target, the rigid transformation between those sets can be solved in a least squares sense with a closed form solution [2. From a statistical point of view, least squares methods assume that the points are observed with isotropic, identical and independent Gaussian noise. A more general approach would be to assume that both the source and the target have noise that can be neither isotropic nor identical. This kind of model leads to a Generalized Total Least Squares (GTLS) problem. Kanatani et al. [3] have introduced a total least squares solution to the problem of rotations of correspondent points.

This paper introduces a novel total least squares generalization of ICP. This generalization will allow us to consider anisotropic noise both in the target shape and the source shape. Moreover, an iterative technique is presented that allows us to estimate the optimal set of covariance matrices so the effect of outlier points is minimized. An evaluation study is carried out to show the superiority of the proposed method compared to the standard ICP. 


\subsection{Iterative Closest Points}

Let $\mathcal{P}$ be the data shape (source shape) and $\mathcal{X}$ the model shape (target shape) to be registered with. The data shape is decomposed in point set form, if not already available in that form. The model shape could originally be given in any representation but for our purpose we will assume that is also decomposed in a point set. Thus, let $N_{P}, N_{X}$ be the number of points in the shapes. $P$ and $X$ are respectively defined by the $N_{P}$-tuple $\mathcal{P}=\left\{\mathbf{p}_{1}, \mathbf{p}_{2}, \cdots, \mathbf{p}_{N_{P}}\right\}$ and the $N_{X}$-tuple $\mathcal{X}=\left\{\mathbf{x}_{1}, \mathbf{x}_{2}, \cdots, \mathbf{x}_{N_{X}}\right\}$. There is not a preestablished correspondence between the points $\mathbf{p}_{j}$ and $\mathbf{x}_{j}$.

ICP finds the rigid body transformation 1 , rotation $\mathbf{R}$ and translation $\mathbf{t}$ that aligns the source with the target by minimizing the distance metric

$$
J(\mathbf{R}, \mathbf{t})=\sum_{i=1}^{N_{P}}\left\|\mathbf{y}_{i}-\mathbf{R} \mathbf{p}_{i}-\mathbf{t}\right\|^{2}, \quad \text { where } \quad \mathbf{y}_{i}=\mathcal{C}\left(\mathbf{R p}_{i}+\mathbf{t}, \mathcal{X}\right)
$$

is a point on the surface of the target model $\mathcal{X}$ that corresponds to the point $\mathbf{p}_{i}$. Given that a priori correspondence between source points and target points does not exist, an iterative search is needed in order to solve the problem. At each iteration, a correspondence is established by means of the correspondence operator $\mathcal{C}$. Then, the transformation that minimizes a mean square metric is computed. The data points are transformed using the computed transformation and the process is repeated until a minimum error threshold is achieved or a maximum number of iterations is reached. The correspondence operator is typically the closest point operator: $\mathcal{C}_{c p}(\mathbf{a}, \mathcal{X})=\arg \min _{\mathbf{x} \in \mathcal{X}}\|\mathbf{x}-\mathbf{a}\|$.

One of the main drawbacks of ICP is that unequal uncertainty among points is not considered in the process. Several authors have tried to partially take into account unequal uncertainty by using a weighted least squares minimization [4] 5.6. This approach, although effective and intuitively correct, is not optimal due to isotropic assumptions.

\section{Method}

Real problems present data with noise both in the source and in the target. Moreover, the noise is typically anisotropic, i.e. certain directions are prone to being more inaccurate than others. These facts lead us to introduce a generalized total least squares approach to the aforementioned registration problem.

\subsection{GTLS Registration of Corresponding 3-D Point Sets}

Let us assume that the target points and the source points are corrupted with additive noise: $\mathbf{x}_{i}=\overline{\mathbf{x}}_{i}+\Delta \mathbf{x}_{i}$ and $\mathbf{p}_{i}=\overline{\mathbf{p}}_{i}+\Delta \mathbf{p}_{i}$, where $\Delta \mathbf{x}_{i}$ and $\Delta \mathbf{p}_{i}$ are independent random variables with zero mean and known covariance matrices

\footnotetext{
${ }^{1}$ The method can also handle affine transformations by looking for the solution in a linear affine subspace
} 
$V\left[\mathbf{x}_{i}\right]=E\left[\Delta \mathbf{x}_{i} \Delta \mathbf{x}_{i}^{T}\right]$ and $V\left[\mathbf{p}_{i}\right]=E\left[\Delta \mathbf{p}_{i} \Delta \mathbf{p}_{i}^{T}\right]$. The relation between the free noise data is given by the rigid transformation model

$$
\overline{\mathbf{x}}_{i}=\mathbf{R} \overline{\mathbf{p}}_{i}+\mathbf{t}, \quad i=1, \cdots, N_{P} .
$$

Our problem is to find an estimator of the rotation matrix, $\hat{\mathbf{R}}$, and the translation term, $\hat{\mathbf{t}}$ from the noisy data $\left\{\mathbf{x}_{i}\right\}$ and $\left\{\mathbf{p}_{i}\right\}$.

The Gauss-Markov theorem states that the linear minimum variance unbiased estimator when the noise is correlated is given by the minimization of the Mahalanobis distance

$$
J(\mathbf{R}, \mathbf{t})=\sum_{i=1}^{N_{P}}\left(\mathbf{p}_{i}-\overline{\mathbf{p}}_{i}\right)^{T} V\left[\mathbf{p}_{i}\right]^{-1}\left(\mathbf{p}_{i}-\overline{\mathbf{p}}_{i}\right)+\sum_{i=1}^{N}\left(\mathbf{x}_{i}-\overline{\mathbf{x}}_{i}\right)^{T} V\left[\mathbf{x}_{i}\right]^{-1}\left(\mathbf{x}_{i}-\overline{\mathbf{x}}_{i}\right),
$$

subject to the model constraint (2). This problem can be seen as a Generalized Total Least Squares problem [7] from the matrix algebra point of view. The main difficulty of this problem is that we are looking for the solution in the group of rotations $S O(3)$. To find a feasible implementation, we have decoupled the problem over the minimization variable. Thus, we will independently minimize the rotation variable and the translation variable. An iterative approach will seek the optimal solution in both spaces.

Rotation term. Let us restrict our data model to be

$$
\overline{\mathbf{x}}_{i}=\mathbf{R} \overline{\mathbf{p}}_{i}, \quad i=1, \cdots, N_{P} .
$$

The minimization of the functional $J$ in eq. (3) subject to (4) has been previously addressed by Ohta and Kanatani [3. The authors show that the values of $\hat{\mathbf{x}}_{i}$ and $\hat{\mathbf{p}}_{i}$ that minimize (3) can be solved analytically using Lagrange multipliers and considering $\mathbf{R}$ fixed. From this solution, the resulting minimum is minimized with respect to $\mathbf{R}$. The problem reduces to

$$
J_{R}(\mathbf{R})=\sum_{i=1}^{N_{P}}\left(\mathbf{x}_{i}-\mathbf{R} \mathbf{p}_{i}\right)^{T} \mathbf{W}_{i}^{\mathbf{R}}\left(\mathbf{x}_{i}-\mathbf{R} \mathbf{p}_{i}\right) \quad \hat{\mathbf{R}}=\arg \min _{\mathbf{R} \in S O(3)} J_{R}
$$

where $\mathbf{W}_{i}^{\mathbf{R}}=\left(\mathbf{R} V\left[\mathbf{p}_{i}\right] \mathbf{R}^{T}+V\left[\mathbf{x}_{i}\right]\right)^{-1}$. The same authors proposed an optimization scheme based on quaternions using the renormalization technique of Kanatani [8]. The implementation of this optimization method has been proven to be robust and stable.

Translation term. In this case, the data model is merely

$$
\overline{\mathbf{x}}_{i}=\overline{\mathbf{p}}_{i}+\mathbf{t}, \quad i=1, \cdots, N_{P}
$$

Following the same approach as in [3], we have solved the values of $\hat{\mathbf{x}}_{i}$ and $\hat{\mathbf{p}}_{i}$ that minimize (3) considering $\mathbf{t}$ fixed. Again, minimizing that result with respect 
to $\mathbf{t}$, we can write

$$
J_{t}(\mathbf{t})=\sum_{i=1}^{N_{P}}\left(\mathbf{x}_{i}-\mathbf{p}_{i}-\mathbf{t}\right)^{T} \mathbf{W}_{i}^{\mathbf{t}}\left(\mathbf{x}_{i}-\mathbf{p}_{i}-\mathbf{t}\right), \quad \hat{\mathbf{t}}=\arg \min _{\mathbf{t} \in R^{3}} J_{t}
$$

where $\mathbf{W}_{i}^{\mathbf{t}}=\left(V\left[\mathbf{p}_{i}\right]+V\left[\mathbf{x}_{i}\right]\right)^{-1} \cdot \hat{\mathbf{t}}$ can be analytically obtained by equating the gradient to zero and solving for $\mathbf{t}$. The solution is given by

$$
\hat{\mathbf{t}}=\left(\sum_{i=1}^{N_{P}} \mathbf{W}_{i}^{\mathbf{t}}\right)^{-1} \sum_{i=1}^{N_{P}} \mathbf{W}_{i}^{\mathbf{t}}\left(\mathbf{x}_{i}-\mathbf{p}_{i}\right) .
$$

Note that setting the covariance matrices to the identity, the solutions $\hat{\mathbf{R}}$ and $\hat{\mathbf{t}}$ reduce to the least squares ones.

Robustness. A total least squares approach has the flexibility of encoding the uncertainty information of the observed data points in the covariance matrices. In point correspondence problems we can identify two sources of noise: extrinsic noise due to sensor errors and intrinsic noise due to incorrect matches. The extrinsic noise is problem dependent and we try to model it by means of the covariance matrices. The intrinsic noise has paramount importance as recognized by Haralick et al. 9]. Outliers make ordinary LS estimators the estimator of least virtue. We have enforced the robustness of the correspondence problem by an iterative algorithm that looks for the optimal set of covariance matrices. At the same time, the iterative approach will allow us to look for the solution in both parameter spaces, rotation and translation.

Our aim is to make the outlier points having a noise covariance higher than initially expected by adding an isotropic part. The covariance matrix can be written as the sum of two terms

$$
V\left[\mathbf{x}_{i}\right]=V_{e x}\left[\mathbf{x}_{i}\right]+\sigma_{x_{i}}^{2} \mathbf{I}_{3} \quad V\left[\mathbf{p}_{i}\right]=V_{e x}\left[\mathbf{p}_{i}\right]+\sigma_{p_{i}}^{2} \mathbf{I}_{3} .
$$

An iterative scheme will look for the optimal set of $\sigma_{x_{i}}^{2}$ and $\sigma_{p_{i}}^{2}$ such that the outliers are identified.

The robust corresponding points algorithm can be summarized as follows:

1. Set initial variable: $\hat{\mathbf{t}}=[0,0,0]^{T}, V\left[\mathbf{x}_{\mathbf{i}}\right]=V_{e x}\left[\mathbf{x}_{\mathbf{i}}\right]+\mathbf{I}, V\left[\mathbf{p}_{\mathbf{i}}\right]=V_{e x}\left[\mathbf{p}_{\mathbf{i}}\right]+\mathbf{I}$.

2. Estimate $\hat{\mathbf{R}}$ as described in [3] using $\left\{\mathbf{x}_{i}^{\prime}=\mathbf{x}_{\mathbf{i}}-\hat{\mathbf{t}}\right\}$ and $\left\{\mathbf{p}_{\mathbf{i}}\right\}$.

3. Rotate source points with estimated $\hat{\mathbf{R}}:\left\{\mathbf{p}_{i}^{\prime}=\hat{\mathbf{R}} \mathbf{p}_{i}\right\}$.

4. Estimate $\hat{\mathbf{t}}$ using eq. (8) with points $\left\{\mathbf{x}_{\mathbf{i}}\right\}$ and $\left\{\mathbf{p}_{i}^{\prime}\right\}$.

5. Update covariance matrix before next iteration

a) $\left\{\mathbf{p}_{i}^{\prime}=\hat{\mathbf{R}} \mathbf{p}_{i}+\hat{\mathbf{t}}\right\}$.

b) Transform source covariance matrix: $V_{e x}\left[\mathbf{p}_{i}\right]=\hat{\mathbf{R}} V_{e x}\left[\mathbf{p}_{\mathbf{i}}\right] \hat{\mathbf{R}}^{T}$.

c) $\sigma_{x_{i}}^{2}= \begin{cases}\left\|\mathbf{p}_{i}^{\prime}-\mathbf{x}_{i}\right\|^{2} / 2 & \text { if } \operatorname{Tr}\left[V_{e x}\left[\mathbf{x}_{i}\right]\right]<3\left\|\mathbf{p}_{i}^{\prime}-\mathbf{x}_{i}\right\|^{2} / 2, \\ 0 & \text { otherwise, }\end{cases}$

$\sigma_{p_{i}}^{2}= \begin{cases}\left\|\mathbf{p}_{i}^{\prime}-\mathbf{x}_{i}\right\|^{2} / 2 & \text { if } \operatorname{Tr}\left[V_{e x}\left[\mathbf{p}_{i}\right]\right]<3\left\|\mathbf{p}_{i}^{\prime}-\mathbf{x}_{i}\right\|^{2} / 2, \\ 0 & \text { otherwise. }\end{cases}$

6. Check for convergence, if not go to step 2. 


\subsection{Generalized Total Least Squares ICP: GTLS-ICP}

The iterative closest points problem defined in section 1.1 can be solved using the minimization method presented in the previous section. In this case, the correspondence between the data points, $\left\{\mathbf{p}_{i}\right\}$, and the model points, $\left\{\mathbf{x}_{i}\right\}$, is not known. For each point, we know the covariance matrix of the noise, $V_{e x}\left[\mathbf{p}_{\mathbf{i}}\right]$ and $V_{e x}\left[\mathbf{x}_{\mathbf{i}}\right]$ respectively. The functional (1) can be rewritten as

$$
J(\mathbf{R}, \mathbf{t})=\sum_{i=1}^{N_{P}}\left(\mathbf{p}_{i}-\overline{\mathbf{p}}_{i}\right)^{T} V\left[\mathbf{p}_{i}\right]^{-1}\left(\mathbf{p}_{i}-\overline{\mathbf{p}}_{i}\right)+\sum_{i=1}^{N_{X}}\left(\mathbf{y}_{i}-\overline{\mathbf{y}}_{i}\right)^{T} V\left[\mathbf{y}_{i}\right]^{-1}\left(\mathbf{y}_{i}-\overline{\mathbf{y}}_{i}\right) .
$$

where $\mathbf{y}_{i}$ is the result of the closest point operator. An iterative closest point approach will start by assuming a matching between data and model points. From this matching, the transformation that minimizes the function (10) will be solved by using the previous algorithm. In the initial iterations, it is dangerous to minimize $J$ using the initial covariances, $V_{e x}$, since the dominant noise will be due to the uncertainty of the matching. Sharp et al. 10] have shown that the noise due to the closest point correspondence operator can be modeled as an isotropic Gaussian noise with variance equal to the distance between the data point and the closest point in the model. This noise model can be introduced to update the covariance matrices globally for each iteration. Thus, the algorithm will initially look for the solution in the least squares sense and the closer the data points and model points get, the more the algorithm will rely on the provided noise covariances, $V_{e x}$.

The GTLS-ICP algorithm can be summarized as follows:

1. Set the initial transformation: $\hat{\mathbf{R}}=\mathbf{I}_{3}, \hat{\mathbf{t}}=[0,0,0]^{T}$.

2. Transform data points: $\left\{\mathbf{p}_{i}^{\prime}=\hat{\mathbf{R}} \mathbf{p}_{i}+\hat{\mathbf{t}}\right\}$.

3. Find closest points: $\left\{\mathbf{y}_{i}=\mathcal{C}_{c p}\left(\mathbf{p}_{i}^{\prime}, \mathcal{X}\right)\right\}$.

4. Noise level estimation due to $\mathcal{C}_{c p}: \sigma^{2}=\frac{1}{N_{P}} \sum_{i=1}^{N_{P}}\left\|\mathbf{y}_{i}-\mathbf{p}_{i}^{\prime}\right\|^{2}$.

5. Update model covariance matrices: $V\left[\mathbf{y}_{i}\right]=V_{e x}\left[\mathbf{y}_{i}\right]+\sigma^{2} \mathbf{I}_{3}$.

6. Solve corresponding points registration between $\left\{\mathbf{y}_{i}\right\}$ and $\left\{\mathbf{p}_{i}\right\} \rightarrow \hat{\mathbf{R}}$ and $\hat{\mathbf{t}}$.

7. Check for convergence, if not go to step 2 .

\section{$3 \quad$ Experiments}

\subsection{Methodology}

The reliability of our algorithm has been tested by random noise simulations. A hip model has been used as the model shape. Points have been randomly sampled from that mesh and transformed with a known rotation matrix $\mathbf{R}$, and translation vector $\mathbf{t}$ (see Fig. (1).

Noise has been independently added to the mesh and the transformed points. The noise between points is independent but not identically distributed with a known covariance matrix. Noise has been generated according to two distributions: Normal and Tukey's slash distribution. The slash distribution can be 


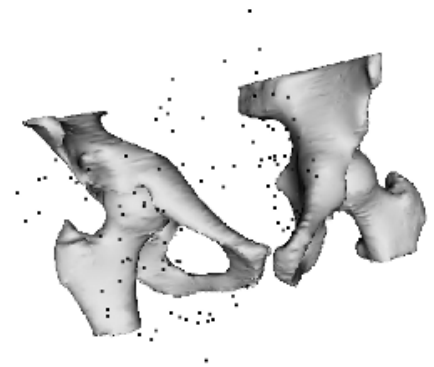

Fig. 1. Hip and random sampled points after transformation used for the evaluation of the algorithm.

Table 1. Eigenvalues of noise covariance matrices for each class. The degree of anisotropy decreases with the the number of the class

\begin{tabular}{c|ccc|cc|ccc||ccc}
\hline Cov. class & $\lambda_{1}$ & $\lambda_{2}$ & $\lambda_{3}$ & Cov. class & $\lambda_{1}$ & $\lambda_{2}$ & $\lambda_{3}$ & Cov. class & $\lambda_{1}$ & $\lambda_{2}$ & $\lambda_{3}$ \\
\hline 1 & 1.00 & 0.00 & 0.00 & 6 & 0.67 & 0.17 & 0.17 & 11 & 0.44 & 0.33 & 0.22 \\
2 & 0.80 & 0.20 & 0.00 & 7 & 0.57 & 0.29 & 0.14 & 12 & 0.40 & 0.40 & 0.20 \\
3 & 0.67 & 0.33 & 0.00 & 8 & 0.50 & 0.38 & 0.12 & 13 & 0.40 & 0.30 & 0.30 \\
4 & 0.57 & 0.43 & 0.00 & 9 & 0.44 & 0.44 & 0.11 & 14 & 0.36 & 0.36 & 0.27 \\
5 & 0.50 & 0.50 & 0.00 & 10 & 0.50 & 0.25 & 0.25 & 15 & 0.33 & 0.33 & 0.33 \\
\hline
\end{tabular}

obtained as a Gaussian random variable with zero mean and a given covariance divided by a uniform random variable over the interval [0,1]. The slash distribution has been used to model outliers due to its broad tails. 15 different classes of noise have been simulated depending on the covariance. Table 1 shows the eigenvalues of the different noise covariance matrices. The first five classes correspond to the case of noise confined onto a plane.

A distance metric on rotation matrices can be defined as the minimal angle of rotation needed, around some axis, to transform one rotation into another. This can be calculated using quaternions of the relative rotation error $\mathbf{R}_{e}=\hat{\mathbf{R}} \mathbf{R}^{T}$. The quaternion representation of $\mathbf{R}_{e}$ is $\mathbf{q}_{e}=\left[q_{0}, q_{1}, q_{2}, q_{3}\right]^{T}$. The error vector is defined as $\Delta \Omega=\Delta \Omega \mathbf{l}_{r}$, where $\Delta \Omega=\arccos \left(2 q_{0}\right)$ is the angle of the rotation error $\mathbf{R}_{e}$ and $\mathbf{l}_{r}=\left[q_{1}, q_{2}, q_{3}\right]^{T} / \sin \left(\frac{\Delta \Omega}{2}\right)$ is the axis of rotation. The variance of the error is given by $V[\Delta \Omega]=E\left\{\Delta \Omega \Delta \Omega^{T}\right\}$.

The two statistics that we have used to assess the rotation error have been the mean rotation error $E_{\Omega}$ and the standard deviation $S_{\Omega}$ defined as

$$
E_{\Omega}=\|E\{\Delta \Omega\}\|, \quad S_{\Omega}=\sqrt{\operatorname{Tr}[V[\Delta \Omega]]} .
$$

Regarding the translation, we have used the mean translation error $E_{t}$ and the standard deviation $S_{t}$ defined as

$$
E_{t}=\|E\{\mathbf{t}-\hat{\mathbf{t}}\}\|, \quad S_{t}=\sqrt{\operatorname{Tr}\left[E\left\{(\mathbf{t}-\hat{\mathbf{t}})(\mathbf{t}-\hat{\mathbf{t}})^{T}\right\}\right]} .
$$

To estimate the error statistics, 100 independent realizations have been carried out for each anisotropic noise class and variance. We have compared our method 
$E_{\Omega}$

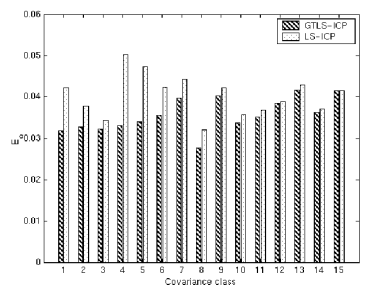

Rotation Error (degrees)

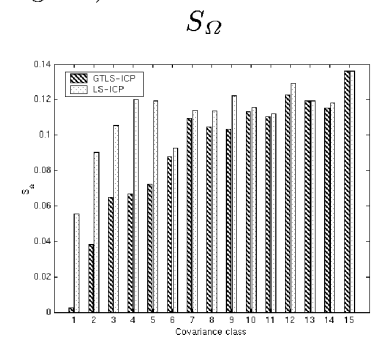

Fig. 2. Evaluation of the rotation error and translation error for different covariance matrices and Gaussian noise. Dark bar: GTLS-ICP. Light bar: LS-ICP

$$
E_{\Omega} \quad \text { Rotation Error (degrees) }
$$

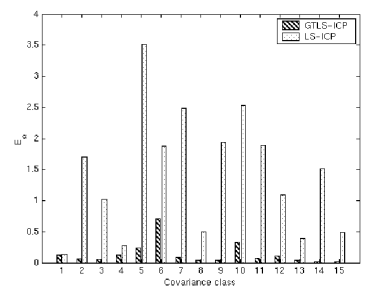

$S_{\Omega}$

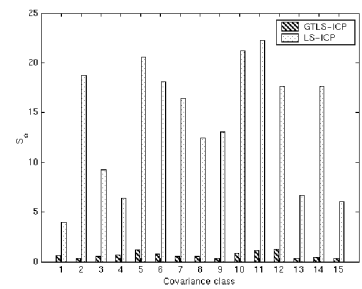

Fig. 3. Evaluation of the rotation error and translation error for different covariance matrices and slash noise. Dark bar: GTLS-ICP. Light bar: LS-ICP.

(GTLS-ICP) with the standard least squares ICP (LS-ICP) using Horn method 2] to minimize the functional at each iteration.

\subsection{Results}

The GTLS-ICP algorithm has been tested by randomly sampling 150 points from the mesh. A rotation of $30^{\circ}$ around the axis $[1,1,1]^{T}$ and a translation $[1,1,1]^{T}$ have been applied. Noise has been added to the mesh and to the sampled points after the transformation.

Figure 2 shows the rotation error statistics for Gaussian noise. From these results we can see that, as long as rotations are involved, the mean error is similar for both methods; however the standard deviation of our solution is significantly smaller for anisotropic noise classes. The more isotropic the noise becomes, our solution tends to the standard least squares as expected. The translation error follows a similar tendency, our method shows a lower $S_{t}$ when the noise distributes in an anisotropic way.

Figure 3 shows the error statistics for slash noise. Slash noise has been only added to the sampled points, while the mesh has been corrupted with Gaussian noise. The results clearly shows the stability and robustness of our method. It is fair to say that a preprocessing for outliers rejection may improve the results for 
LS-ICP. However, our method seamlessly incorporates the rejection of outliers by setting up the covariance matrices adequately.

\section{Conclusions}

A generalization of the iterative closest points algorithm has been presented using a generalized total least squares framework. The main contributions of this work are: 1) noise is modeled both in the target shape and the source shape (total least squares), 2) anisotropic noise can be taken into account in the estimation of the transformation (generalized total least squares) and 3) outlier rejection is intrinsically handled by iterative estimation of the optimal isotropic noise variance of the covariance matrices. We believe that registration techniques that use ICP, or some of its variants, as optimization process will directly benefit of the generalized approach introduced in this paper, yielding a more robust estimation.

Acknowledgments. This work has been funded by CIMIT and NIH grant P41 RR 13218.

\section{References}

1. Paul J. Besl and Neil D. McKay. A method for registration of 3-D shapes. IEEE Transaction on Pattern Analysis and Machine Intelligence, 14(2):239-256, 1992.

2. B. K. P. Horn. Closed-form solution of absolute orientation using unit quaternions. Journal of the Optical Society of America, 4(4):629-642, 1987.

3. N. Ohta and K. Kanatani. Optimal estimation of three-dimensional rotation and reliability evaluation. IEEE Transactions on Information and Systems, E81D(11):1247-1252, 1998.

4. Zhengyou Y. Zhang. Iterative point matching for registration of free-form curves and surfaces. International Journal of Computer Vision, 13(2):119-152, Oct 1994.

5. Calvin R. Maurer, Georges B. Aboutanos, Benoit M. Dawant, Robert J. Maciunas, and J. Michael Fitzpatrick. Registration of 3-D images using weighted geometrical features. IEEE Transaction on Medical Imaging, 15(6):836 - 849, Dec 1996.

6. Chitra Dorai, John Weng, and Anil K. Jain. Optimal registration of objects view using range data. IEEE Transaction on Pattern Analysis and Machine Intelligence, 19(10):1131 - 1138, Dec 1997.

7. S. van Huffel and J. Vandewalle. Analysis and properties of the generalized total least squares problem $A X \approx B$, when some or all columns are subject to error. SIAM Journal on Matrix Analysis and Applications, 10(3):294 - 315, 1989.

8. K. Kanatani. Statistical Optimization for Geometric Computation: Theory and Practice. Elsevier, 1996.

9. Robert M. Haralick, Hyonam Joo, Chung-Nan Lee, Xinhua Zhuang, Vinay G. Vaidya, and Man Bae Kim. Pose estimation from corresponding point data. IEEE Transactions on Systems, Man. and Cybernetics, 19(6):1426 - 1446, Nov 1989.

10. Gregory C. Sharp, Sang W. Lee, and David K. Wehe. ICP registration using invariant features. IEEE Transaction on Pattern Analysis and Machine Intelligence, 24(1):90-102, Jan 2002. 